\title{
A Policy for Open-Loop Attenuation of Disturbance Effects Caused by Uncertain Ground Properties in Running
}

\author{
Jonathan W. Hurst \\ The Robotics Institute \\ Carnegie Mellon University \\ Pittsburgh, Pennsylvania \\ jhurst@ri.cmu.edu
}

\author{
Benjamin Morris \\ EECS Department \\ University of Michigan \\ Ann Arbor, Michigan \\ morrisbj@umich.edu
}

\author{
Joel E. Chestnutt \\ The Robotics Institute \\ Carnegie Mellon University \\ Pittsburgh, Pennsylvania \\ chestnutt@ri.cmu.edu
}

\author{
Alfred A. Rizzi \\ The Robotics Institute \\ Carnegie Mellon University \\ Pittsburgh, Pennsylvania \\ arizzi@bostondynamics.com
}

\begin{abstract}
Outside of the laboratory, accurate models of ground impact dynamics are either difficult or impossible to obtain. Instead, a rigid ground model is often used in gait and controller design, which simplifies the system model and allows attention to remain focused on other aspects of running. In real-world terrain this simplification may overlook important dynamic effects. Immediately following a foot touchdown event, sensitivity to ground stiffness is at its highest and at the same time the accuracies of state estimates are at their lowest. Even if ground stiffness is known and state estimates are accurate, actuator bandwidth limitations make immediate compensation difficult. Taking inspiration from nature, we propose a novel solution to attenuate the effects of unexpected ground stiffness changes using a unified control system comprised of hardware passive dynamics and open-loop software control policies.
\end{abstract}

\section{INTRODUCTION}

As humans walk or run across tile, concrete, grass, sand and any number of other surfaces, we unconsciously adjust leg stiffness to compensate for significant variations in ground stiffness. A person running in the dark lacks visual reference to ground changes, yet can often run without falling. In general, animals (including bipeds) are exceptionally good at running robustly over rough terrain, rarely stumbling even at high speeds. Intuitively we recognize that animals cannot be controlling the precise toe position or the toe forces at ground impact. For animals to exhibit such stability, passive dynamic effects that help attenuate ground uncertainty are likely to exist, in addition to the obvious stabilizing effect of neuromuscular control. Indeed, in tests where human subjects hop on surfaces that change stiffness unexpectedly, a compensating leg stiffness adjustment is measured that occurs more quickly than would be possible with conscious or reflexive neural responses [1], further supporting the existence of passively stabilizing effects resulting from the human morphology.

Taking inspiration from nature, we propose a method for attenuating ground uncertainties in robotic running that is loosely based on pre-activation of muscles and the energystoring properties of tendons. Arguing that the developed policy corresponds with the strategy actually employed by animals is beyond the scope of this paper. However, we demonstrate through simulation that a physical leg spring, combined with an open-loop time-based spring set point trajectory that is triggered at ground impact, results in

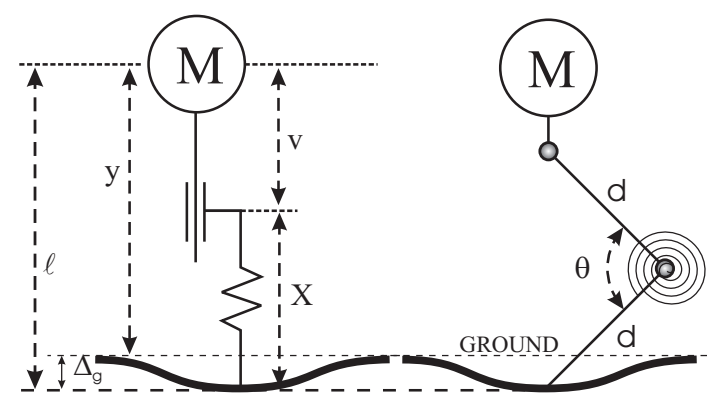

Fig. 1. Left: The spring-mass model, constrained to hop vertically. The mass and leg spring stiffness are constants, but the position of the spring set point, labeled $v$ in the figure, can be controlled. The length of the leg spring is represented as $X$. The overall leg length is represented as $(X+$ $v=\ell$ ). The ground surface is compliant, and deflects under the force applied by the leg spring by an amount $\Delta_{g}$. The position of the center of mass relative to the undeflected ground surface is labeled $y$. Right: The basic Spring Loaded Inverted Pendulum model, modified with a knee and rotational spring, constrained to hop vertically.

robustness against ground stiffness uncertainty in the first portions of stance, during which software control is relatively ineffective. After the transient dynamics of impact have passed, and for the rest of the duration of stance, a software controller can influence the behavior of the system.

The rationale for seeking a passively stabilizing effect may not be obvious. In robots with sufficient actuation, a computer control system can control most of the high-level behavior of a robot. However, in these same robots, passive stabilization becomes important when the time scale of a perturbation is sufficiently fast that the software control system cannot influence the robot dynamics quickly enough. In animals, neural delay may be the limiting factor which determines which aspects of control are better handled passively, rather than through active control. In robots, computational delay (perhaps due to sensor filtering) or actuator limitations (perhaps due to the band-limiting effects of rotor inertia on torque delivery) are more likely to limit the effectiveness of software control. For example, during toe impact, the software has little effect on the instantaneous response of the system. The instantaneous response will be a function of the leg stiffness, toe mass, and other physical properties.

Theories regarding passive stability effects are, by necessity, based on simplified models. A one-dimensional spring- 
mass model, shown in Figure 1, is the simplest model that can be used to illustrate the ideas of this paper. The 1DOF model is a reduction of the $2 \mathrm{DOF}$ model more commonly used for animal gait analysis [2]. These spring-mass models are derived from biomechanical evidence that animals utilize physical springs to store and release potential and kinetic energy from the flight phase of a running gait [3]. The repetitive motion of the center of mass forms a limit cycle, with stability of the running gait corresponding to stability of the limit cycle. By developing a clear understanding of the interactions between hardware mechanism design and software control system design and the limitations of each, we believe that many disturbances, including ground stiffness disturbances, can negotiated without the use of excessive control effort.

\section{BACKGROUND}

Substantial prior research inspires and supports our model, our simplifying assumptions, and our hypothesis. Our springmass model is based on an approximation used to describe the center of mass motion of a running animal [4]. This behavior is partly implemented through the use of natural dynamics of animal physiology; certain muscles and tendons in animals function as large springs acting in series with actuators [5], [6], [7]. Although tendons store the majority of the spring energy, muscle trajectories also influence a leg's spring-like behavior, and animals adjust their muscle trajectories to exhibit different spring-like behaviors [8]. Leg stiffness can change quickly-experiments have shown that humans adjust their leg stiffness to accommodate an unexpected change in ground stiffness within a single stride [9]. Similar experiments demonstrated that humans can change their leg stiffness faster than any possible neural response [1]. This may be explained in part by the experimental observation that some muscle behavior is determined by pre-planned trajectories and not by reflex or other sensory feedback [10], [11]. In addition to muscle behavior, leg geometry contributes to the overall spring-like behavior in humans [12]. Test subjects that hop on a springy surface increase leg stiffness by landing with straighter knees, and/or by utilizing muscle activation to increase joint stiffness.

Thus far, references have suggested that animals utilize pre-planned, open-loop muscle trajectories for some aspects of running or hopping gaits. It may seem that open-loop behavior is less than ideal, but in a simulation study by Kubow et al., a simplified planar model of cockroaches with open-loop muscle trajectories showed stable behavior[13]. This is a good example of open-loop cyclic stability effects, although it may be advantageous to include feedback control when possible. This paper in particular is in agreement with our philosophical approach, especially the introductory quote from Raibert and Hodgins [14]:

"Many researchers in neural control think of the nervous system as a source of commands that are issued to the body as direct orders. We believe that the mechanical system has a mind of its own, governed by the physical structure and laws of physics. Rather than issuing commands, the nervous system can only make suggestions which are reconciled with the physics of the system and task at hand."

\section{HYPOTHESIS}

As discussed in the Background section, humans can adjust leg stiffness faster than any neural responses. Our most plausible hypothesis to explain this phenomenon suggests that muscles (analogous to the spring set point) begin movement with a pre-planned time-based trajectory, triggered by the anticipated ground contact, and calculated for a specific ground stiffness. The time-based muscle trajectory acts in series with the springy tendons of the leg to create an overall spring-like leg behavior. However, because the muscle trajectory is based only on time rather than on applied external forces like the springy tendon, the leg system changes its behavior based on the rate of applied ground force at the toe. This change in behavior causes the leg to effectively "stiffen" after landing on soft surfaces, and causes the leg to effectively "soften" after landing on hard surfaces. In other words, the actuation trajectory along with the series spring may create a mechanical feedback mechanism that has a stabilizing effect on the limit cycle of running. The effects are only important during the first instants of stance, since after some time, the software can effectively control the muscle trajectory using sensory feedback.

A similar stabilizing effect is reproduced in the 1DOF hoppers of Fig. 1, when an open-loop motor trajectory is used in series with a physical leg spring to simulate the action of a spring that is stiffer than the one physically present. Stated differently, the presence of a soft spring in conjunction with a set point trajectory that is compressing the spring on impact will attenuate the destabilizing effects of ground stiffness uncertainty.

\section{Hardware ANd Control Design Policy}

In this section we explain the basic spring-mass models of Figure 1 and some necessary simplifying assumptions. Next, we derive the dynamics of two hoppers, one with a linear prismatic leg spring and the other with a linear rotational spring at the knee. From these equations, openloop controllers are derived that modify spring set points in order to change the effective spring stiffness. We explain in words the short-term stabilizing effects of these open loop controllers, and lastly, discuss the stabilizing effects of having a knee versus having a prismatic leg spring.

\section{A. Model and Assumptions}

The leg is a series chain of the following parts: a mass (representing the body of a animal), a spring set point adjustment (representing the net effect of leg muscle activation), a spring (representing the net compliance of leg muscles and tendons), and a linearly compliant ${ }^{1}$ ground surface. The leg

\footnotetext{
${ }^{1}$ Representing the ground as a massless linear spring is a major simplification of natural ground properties. This linear model is adequate for the purposes of this initial study and could be considered a limiting case.
} 
may or may not have a knee, see Figure 1. In either case, the mass is constrained to travel only on the vertical axis. All components of the model are massless except for the body, and as such, the entire model has only a single degree of freedom, which is the height of the center of mass. This degree of freedom is controlled by moving the spring set point, so that the behavior of the leg is governed by more than merely the properties of the passive spring.

\section{B. System Dynamics with or without a Knee}

With or without a knee, the vertical acceleration of the center of mass of the robot, $\ddot{y}$, can be found by summing the forces at this point, resulting in

$$
\ddot{y}=-g+\frac{F_{y}}{m},
$$

where $g$ is the magnitude of the acceleration of gravity and $F_{y}$ is the magnitude of the force exerted in the vertical direction by the prismatic or rotational spring.

For the hopper with a prismatic leg spring,

$$
F_{y}=\mathbf{K}_{\mathrm{pr}}\left(\ell_{0}-\ell\right),
$$

where $\mathbf{K}_{\mathrm{pr}}>0$ is a spring constant, $\ell_{0}$ is the rest length of the leg (the distance from toe to body when no toe force is applied), and $\ell$ is the instantaneous length of the leg. Assuming the surface of the ground is purely elastic and also massless, the restoring forces of the ground must match the forces exerted by the prismatic leg spring, so that

$$
\mathbf{K}_{\mathrm{pr}}\left(\ell_{0}-\ell\right)=\mathbf{K}_{\mathrm{g}} \Delta_{g},
$$

where $\mathbf{K}_{\mathrm{g}}>0$ is the ground stiffness constant and $\Delta_{g}$ is the ground deflection measured vertically at the point of contact with the toe. From the geometry of Fig. 1,

$$
\ell=y+\Delta_{g} .
$$

Using (2) (3) and (4), the force $F_{y}$ is found in terms of $y$ and $\ell_{0}$,

$$
F_{y}=\frac{\mathbf{K}_{\mathrm{g}} \mathbf{K}_{\mathrm{pr}}}{\mathbf{K}_{\mathrm{g}}+\mathbf{K}_{\mathrm{pr}}}\left(\ell_{0}-y\right) .
$$

Equation (1) can now be rewritten as

$$
\ddot{y}=-g+\frac{1}{m}\left(\frac{\mathbf{K}_{\mathrm{g}} \mathbf{K}_{\mathrm{pr}}}{\mathbf{K}_{\mathrm{g}}+\mathbf{K}_{\mathrm{pr}}}\right)\left(\ell_{0}-y\right),
$$

which gives the acceleration of the center of mass $\ddot{y}$ as a function of the height of the center of mass $y$ and the position of the prismatic spring set point $\ell_{0}$. For a fixed $\ell_{0}$ and known initial condition $\left(t_{0}, y_{0}, \dot{y}_{0}\right)$, the above can be solved for $y(t)$ giving

$$
\begin{aligned}
y(t)=\frac{\dot{y}_{0}}{\sqrt{\alpha_{1}}} \sin \left(\sqrt{\alpha_{1}}\left(t-t_{0}\right)\right)+ \\
\quad\left(y_{0}-\frac{\alpha_{2}}{\alpha_{1}}\right) \cos \left(\sqrt{\alpha_{1}}\left(t-t_{0}\right)\right)+\frac{\alpha_{2}}{\alpha_{1}}
\end{aligned}
$$

where $\alpha_{1}=\frac{1}{m}\left(\frac{\mathbf{K}_{\mathrm{g}} \mathbf{K}_{\mathrm{pr}}}{\mathbf{K}_{\mathrm{g}}+\mathbf{K}_{\mathrm{pr}}}\right)$ and $\alpha_{2}=-g+\alpha_{1} \ell_{0}$.
The dynamics of a robot with a rotational knee spring can be similarly derived, but with equations (2) and (3) replaced by

$$
F_{y}=\mathbf{K}_{\text {rot }}\left(\theta_{0}-\theta\right) d \cos (\theta / 2)
$$

and

$$
\begin{aligned}
\ell & =2 d \sin (\theta / 2)=y+\Delta_{g} \\
\ell_{0} & =2 d \sin \left(\theta_{0} / 2\right),
\end{aligned}
$$

which are each based on the geometry of a hopper with a knee spring of stiffness $\mathbf{K}_{\text {rot }}>0$. In the general case $\theta$ cannot be explicitly found in terms of $y, F_{y}$, and $\theta_{0}$ and thus a simple closed form solution of the dynamics is not readily available. Instead numerical integration is used to find trajectories of the hopper with a knee.

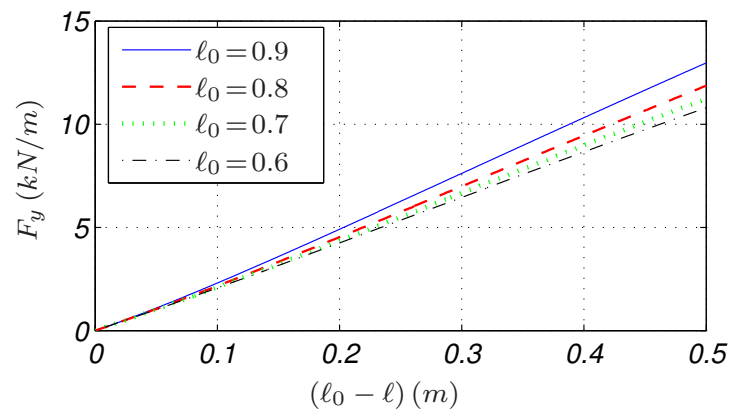

Fig. 2. Vertical toe force, $F_{y}$, as a function of leg deflection, $\left(\ell_{0}-\ell\right)$, for a linear rotational knee spring. Although the function appears quite linear for a particular spring set point, $\ell_{0}$, the stiffness does change for different values of $\ell_{0}$. For the purposes of our simplified simulation, this is the primary difference between a rotational knee spring and a prismatic leg spring.

\section{An Open Loop Control Policy}

In the absence of disturbances and modeling error, it is possible to derive an open loop trajectory for the spring set point that will result in an effective change in spring stiffness.

In the case of the hopper with a prismatic leg spring, define the global stiffness as the net stiffness of the leg in series with the ground, which is equal to

$$
\mathbf{K}_{\text {global }}=\frac{\mathbf{K}_{\mathrm{g}} \mathbf{K}_{\mathrm{pr}}}{\mathbf{K}_{\mathrm{g}}+\mathbf{K}_{\mathrm{pr}}} .
$$

Define a desired global stiffness as

$$
\mathbf{K}_{\text {global,des }}=\frac{\mathbf{K}_{\mathrm{g}} \mathbf{K}_{\mathrm{pr}, \mathrm{des}}}{\mathbf{K}_{\mathrm{g}}+\mathbf{K}_{\mathrm{pr} \text {,des }}} .
$$

for a desired stiffness of the leg spring, $\mathbf{K}_{\mathrm{pr} \text {,des }}>0$.

The control policy is applied to the spring set point, $\ell_{0}$. It is an open loop, time based trajectory triggered by toe contact with the ground, which indicates the beginning of the stance phase. The purpose of the controller is to change the effective leg stiffness, causing the nominal system

$$
\ddot{y}=-g+\frac{\mathbf{K}_{\text {global }}}{m}\left(\ell_{0}-y\right)
$$

with a moveable spring setpoint $\ell_{0}$ to behave like the desired system

$$
\ddot{y}=-g+\frac{\mathbf{K}_{\text {global,des }}}{m}\left(\ell_{0}^{*}-y\right)
$$


with a fixed spring setpoint $\ell_{0}^{*}$. To achieve matching, set

$$
\mathbf{K}_{\text {global,des }}\left(\ell_{0}^{*}-y\right)=\mathbf{K}_{\text {global }}\left(\ell_{0}-y\right),
$$

and solve for the spring set point $\ell_{0}$ as a function of time. Because the control law is designed to be open loop (not require measurements of any state), set $y=y^{*}(t)$, where $y^{*}(t)$ is the solution (7) with $\alpha_{1}=\mathbf{K}_{\text {global,des }} / m$ and $\alpha_{2}=-g+\alpha_{1} \ell_{0}^{*}$. The resulting open loop controller is

$$
\ell_{0}(t)=\left(1-\frac{\mathbf{K}_{\text {global,des }}}{\mathbf{K}_{\text {global }}}\right) y^{*}(t)+\left(\frac{\mathbf{K}_{\text {global,des }}}{\mathbf{K}_{\text {global }}}\right) \ell_{0}^{*} .
$$

Derived by a similar procedure for the hopper with a knee, the open loop set point control policy that results in a change in effective spring stiffness is

$$
\theta_{0}(t)=\theta^{*}(t)-\left(\frac{F_{y}^{*}(t)}{\mathbf{K}_{\mathrm{rot}} d \cos \left(\theta^{*}(t) / 2\right)}\right),
$$

where $F_{y}^{*}(t)$ and $\theta^{*}(t)$ are the force and angle profiles obtained by numerical integration from the system

$$
\ddot{y}=-g+\frac{\mathbf{K}_{\text {rot }, \operatorname{des}}\left(\theta_{0}^{*}-\theta\right) d \cos (\theta / 2)}{m},
$$

initialized at the touchdown time $t_{0}$.

\section{Remarks}

We now have notation for the variables of our spring-mass model defined in Section IV-B, so our hypothesis can be stated in greater detail. We hypothesize that a muscle (spring set point) trajectory, $\ell_{0}(t)$, is initiated upon toe contact with the ground. This muscle trajectory is pre-computed, based on the expected properties of the ground (simplified to a ground stiffness $\mathbf{K}_{\mathrm{g}}$ in our derivations), and it is initiated at ground contact either by anticipation of contact or by a physical trigger. The leg spring stiffness $\mathbf{K}_{\mathrm{pr}}$ is softer than the desired leg stiffness $\mathbf{K}_{\mathrm{pr} \text {,des}}$, but the set-point trajectory $\ell_{0}(t)$ causes the spring to exert forces as if it were the correctly tuned spring. In other words, as the spring compresses due to ground forces, the spring set point is advancing, forcing the spring to compress further.

If the ground is exactly the stiffness that is expected, then the combined behaviors of the leg spring and the spring set-point trajectory $\ell_{0}(t)$ result in the correct desired leg

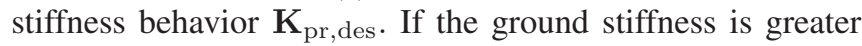
than expected, the leg spring will deflect at a faster rate than anticipated. The spring set point trajectory $\ell_{0}(t)$ is timebased and thus completely unaffected by ground stiffness changes, so it does not deflect faster along with the spring. Thus, for a particular leg length, the set point deflection is a smaller portion of the overall leg deflection, while the spring is a greater portion. The force being applied by the leg at its current position is lower than it would be if the leg had taken longer to reach its current position and allowed the set point trajectory to compress the spring further. In effect, the stiffness of the leg has been reduced.

The effect is similar for a disturbance in the opposite direction, landing on ground that is softer than expected. The leg will deflect at a lower rate than normal, while the pre-planned trajectory $\ell_{0}(t)$ moves forward as planned. At a particular leg deflection, the leg will be applying more force than it would in the normal situation, and the stiffness of the leg has effectively been increased.

\section{E. Adding a Knee}

While this stabilizing effect works for a prismatic spring in series with a set point, the effect is amplified by adding a knee. This change of kinematics adds some complexity to our model; rather than a basic vertical pogo stick, the system is now a mass on top of a single degree of freedom leg that uses a knee rather than a prismatic joint. The hopper is still constrained to vertical hopping, the leg is still massless. The spring is now a rotational spring at the knee rather than a prismatic spring along the length of the leg. The rotational spring is in series with a rotational motor, such that deflection of the leg can be caused by deflection of the knee spring, motion of the motor, or both. In this single degree of freedom system, a knee is essentially a linkage that translates the rotational knee motion to a vertical toe motion, with a nonlinear softening mechanical advantage governed by the sine of the knee angle.

By adding a knee, the spring behavior is determined not only by the position of the set point and the deflection of the spring at a particular time, but also by the orientation of the knee relative to the deflection of the spring. Consider the scenario in which the leg is mostly folded, and the spring is undeflected; in other words, the motor has rotated the knee to a mostly folded position, with no external load. In this scenario, the effective vertical leg spring will be much softer than with a straighter knee. Conversely, a nearly straight leg will produce a high effective vertical stiffness.

When the robot or animal is hopping and the foot hits softer ground, the leg will compress more slowly than expected, and the pre-planned trajectory will begin compressing the knee spring on schedule. However, because the leg will be straighter than expected, the vertical force on the ground will be higher for a given spring deflection. Thus, the knee joint is accentuating the already existing stability effect, and the leg spring is essentially behaving like a stiffer spring, partially compensating for the softness of the ground.

\section{Simulation}

Based on the equations of Section IV-B, a simulator was programmed using Matlab's ODE45 command and used to test the behavior of the system. Parameters for simulation, including initial conditions, are shown in Table I. In each case, simulations were initialized with the robot in flight.

Figure 3 plots effective leg stiffness as the robot encounters three different ground types. In each case, the desired leg stiffness $\mathbf{K}_{\mathrm{pr} \text {,des }}$ is $20 \mathrm{kN} / \mathrm{m}$ and the actual leg stiffness $\mathbf{K}_{\mathrm{pr}}$ is $10 \mathrm{kN} / \mathrm{m}$. The expected ground stiffness $\mathbf{K}_{\mathrm{g}}$ is $50 \mathrm{kN} / \mathrm{m}$. Spring set points are modulated using the open-loop policy of (15).

Effective leg stiffness is defined as the total force applied 
TABLE I

PARAMETERS OF THE SPRING MASS MODEL.

\begin{tabular}{|c|c|c|}
\hline Parameter & Units & Value \\
\hline Rest length of prismatic leg: $\ell_{0}$ & $\mathrm{~m}$ & 0.75 \\
\hline Length of thigh and shin links: $d$ & $\mathrm{~m}$ & 0.5 \\
\hline Body mass: $m$ & $\mathrm{~kg}$ & 40 \\
\hline Expected ground stiffness: $\mathbf{K}_{\mathrm{g}}$ & $\mathrm{kN} / \mathrm{m}$ & 50 \\
\hline Actual ground stiffness: $\mathbf{K}_{\mathbf{g} \text {, act }}$ & $\mathrm{kN} / \mathrm{m}$ & varies \\
\hline $\begin{array}{l}\text { Desired spring stiffnesses: } \\
\mathbf{K}_{\mathrm{pr} \text {,des }}, \mathbf{K}_{\text {rot,des }}\end{array}$ & $\mathrm{kN} / \mathrm{m}, \mathrm{kN} / \mathrm{rad}$ & 20,40 \\
\hline $\begin{array}{l}\text { Actual spring stiffnesses: } \\
\mathbf{K}_{\mathrm{pr}}, \mathbf{K}_{\text {rot }}\end{array}$ & $\mathrm{kN} / \mathrm{m}, \mathrm{kN} / \mathrm{rad}$ & varies, varies \\
\hline Acceleration due to gravity: $g$ & $\mathrm{~m} / \mathrm{s}^{2}$ & 9.81 \\
\hline
\end{tabular}

to the ground by the spring, divided by the net leg deflection:

$$
\mathbf{K}_{\mathrm{pr}, \mathrm{eff}}(t)=\frac{F_{y}(t)}{\ell_{0}^{*}-\ell(t)} .
$$

As seen in Figure 3, the effective leg stiffness is higher in response to lower ground stiffness and lower in response to higher ground stiffness. In the absence of disturbances, that is, when the ground stiffness is as expected, the effective leg stiffness is equal to the desired leg stiffness. Thus, the open-loop spring set point trajectory causes the leg stiffness to compensate for changes in ground stiffness, even when neither is explicitly measured.

Figure 4 explains the open-loop stiffness adjustment seen in Figure 3. When ground stiffness is as expected, the spring compresses and relaxes in a trajectory that complements the spring set point trajectory $\ell_{0}(t)$, and results in a spring-like behavior identical to the desired leg spring $\mathbf{K}_{\mathrm{pr} \text {,des }}$. When ground is softer than expected, the leg spring takes longer to deflect, while the set point trajectory is independent of ground stiffness. In comparison to landing on ground of the expected stiffness, identical leg deflections include higher spring deflections, resulting in higher forces, meaning that effective leg stiffness is higher.

Figure 5 shows how changes in effective stiffness provide open-loop stabilizing effects in the first portions of the stance phase, with benefits quantified in Figure 6.

\section{CONCLUSIONS AND Discussion}

Throughout the paper, we have talked about ground stiffness as an ideal linear spring. A linear stiffness is a poor approximation of dynamic ground properties, as is a linear viscous damping. However, it should not affect the stabilizing effects described in this paper. If the ground gives way more quickly than expected at the beginning of stance, the leg stiffens; if the ground does not give way as fast as expected, then the leg softens. Even for complex dynamic ground behavior, the leg stiffness adjustment will attenuate the effects of a change in ground properties on the center of mass motion of a running robot.

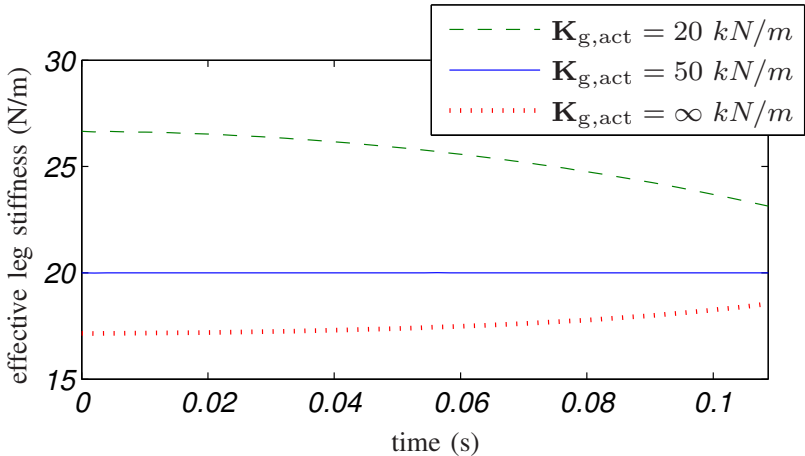

Fig. 3. The effective leg stiffness, $\mathbf{K}_{\mathrm{pr} \text {,eff }}$, as a function of time for three different ground stiffnesses. Each line results from the same pre-planned set point trajectory $\ell_{0}(t)$ and the same physical leg stiffness $\mathbf{K}_{\mathrm{pr}}=10 \mathrm{kN} / \mathrm{m}$, but a different ground stiffness $\mathbf{K}_{\mathrm{g} \text {,act }}$. When the expected ground stiffness of $\mathbf{K}_{\mathrm{g}}=50 \mathrm{kN} / \mathrm{m}$ is encountered (the case of no disturbance), the effective leg stiffness is equal to the desired leg stiffness, $\mathbf{K}_{\mathrm{pr} \text {,des }}=20 \mathrm{kN} / \mathrm{m}$, as depicted by the solid horizontal line. The effective leg stiffness increases when the ground stiffness is lower than expected, and vise-versa.

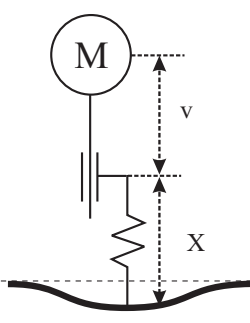

ground as expected

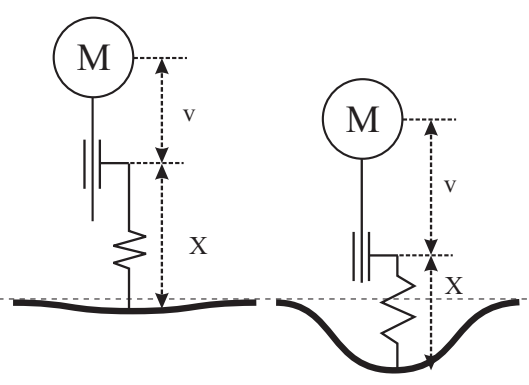

ground too stiff ground too soft
Fig. 4. These figures are snapshots of the spring-mass system hopping on three different ground stiffnesses, shown at the same leg deflection, which occurs at different times. The leg spring has deflected a different amount in each figure, and thus is applying a different force on the ground in each figure. A different force at a particular deflection corresponds with a different leg stiffness.

For variations in ground height rather than variations in ground properties, the result is somewhat different than the stabilizing effect described in this paper. An increase in ground surface height causes an early toe contact, and the soft leg spring begins deflecting before the pre-planned set point moves; thus, the overall leg stiffness is reduced, and the mechanical advantage of the knee accentuates the effect. Conversely, a decrease in ground surface causes late toe contact, such that the pre-planned trajectory extends the leg under no spring load, straightening the knee, before the toe contacts the ground. Thus, the overall leg stiffness increases. We have not simulated or explored the effects on the center of mass trajectory due to the changing leg stiffness on ground height variations, though it will be interesting for future work.

This research is fundamentally different from central pattern generators (CPGs) or open-loop trajectories for general control, like the stable planar cockroach simulation described in the background section. We are considering the time-based 

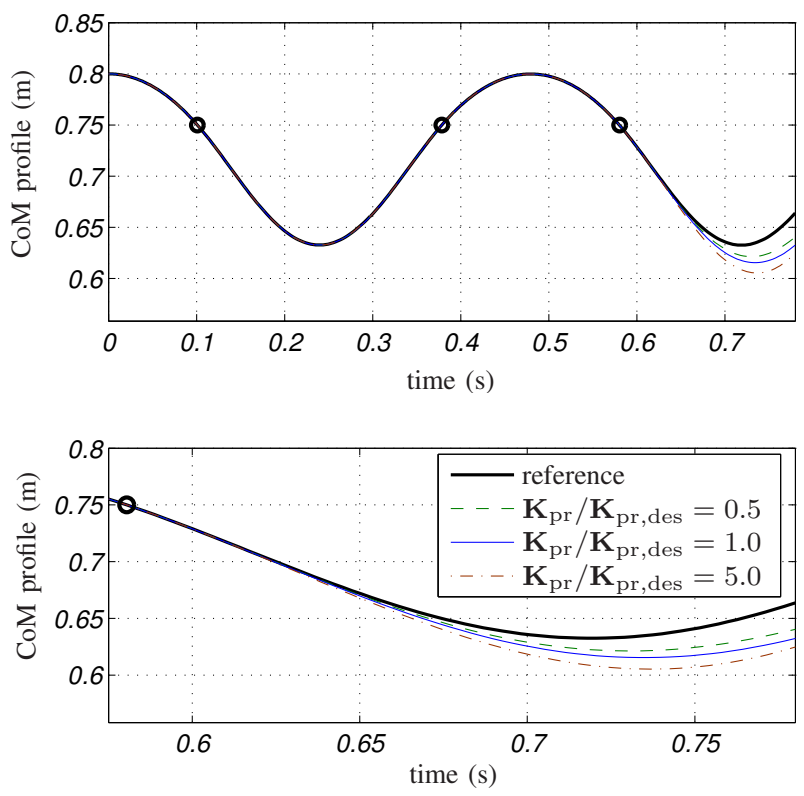

Fig. 5. Top: The position of the center of mass of our spring-mass model as a function of time. Bottom: detail of Top. Values above $0.75 \mathrm{~m}$ correspond to the flight phase, values below $0.75 \mathrm{~m}$ correspond to the stance phase. At approximately $0.5 \mathrm{~s}$, the ground stiffness changes from the expected $50 \mathrm{kN} / \mathrm{m}$ to an unanticipated $30 \mathrm{kN} / \mathrm{m}$. The solid bold line is a reference trajectory, showing the center of mass trajectory in the absence of any disturbance. Each of the other lines shows the center of mass trajectory for different physical leg stiffnesses encountering the ground stiffness disturbance. The softer the leg spring, the lesser the disturbance.

open-loop operation only during the initial instants of stance, because these first instants are when sensor delay, inertias, and computational delay prevent the software from exerting control over the mechanical system. At first impact, with mechanical stabilizing effects, the system begins corrective action instantaneously. After some amount of time, the computer can accurately sense the new disturbances, calculate corrective actions, and command the motor; the motor can then begin accelerating its rotor mass and move to a position or velocity to implement more complex corrective actions than are possible through natural dynamics.

We have shown an open-loop stabilizing effect for running that is implemented through a combination of time-based trajectory and passive spring, and is accentuated by using a knee rather than a prismatic leg. The idea for these effects is inspired by results from the field of biomechanics, but validated in a mathematical simulation. Our opinion is that this effect will be useful for running robots, but it depends highly on the actuator limitations and the type of terrain. The importance of these open-loop stabilizing effects for running robots will be determined when they are examined in the context of a specific robot.

\section{ACKNOWLEDGEMENTS}

This work is supported in part by an NSF IGERT traineeship, award number 0333420, held by the first author. The work of Benjamin Morris is supported by NSF grant ECS 0600869 .

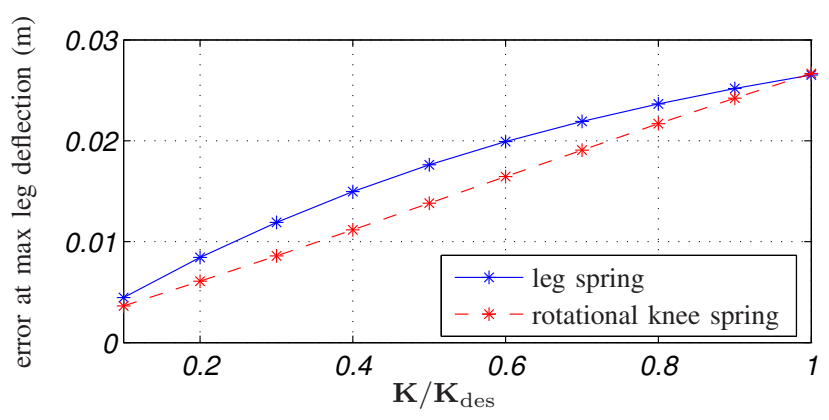

Fig. 6. Decreasing actual leg stiffness $\mathbf{K}$, while holding desired leg stiffness $\mathbf{K}_{\text {des }}$ constant results in lower sensitivity to ground stiffness changes, to the limit where zero leg stiffness shows no effect from ground stiffness changes. Data points are calculated by measuring the maximum disturbance to the center of mass motion due to a ground stiffness change. Actual ground stiffness $\mathbf{K}_{\mathrm{g} \text {,act }}=20 \mathrm{kN} / \mathrm{m}$ and expected ground stiffness $\mathbf{K}_{\mathrm{g}}=50 \mathrm{kN} / \mathrm{m}$ are the same for each data point, only leg stiffness changes.

\section{REFERENCES}

[1] C. T. Moritz and C. T. Farley, "Passive dynamics during leg change mechanics for an unexpected surface during human hopping," Journal of Applied Physiology, vol. 97, pp. 1313-1322, May 2004.

[2] R. Blickhan, "The spring-mass model for running and hopping," Journal of Biomechanics, vol. 22, no. 11/12, pp. 1217-1227, 1989.

[3] G. A. Cavagna, "Elastic bounce of the body," Journal of Applied Physiology, vol. 29, no. 3, pp. 279-282, 1970.

[4] R. Blickhan and R. J. Full, "Similarity in multilegged locomotion: Bouncing like a monopode," Journal of Comparative Physiology, pp. 509-517, 1993.

[5] T. J. Roberts, "The integrated function of muscles and tendons during locomotion," Comparative Biochemistry and Physiology part A, pp. 1087-1099, 2002.

[6] G. A. Cavagna, N. C. Heglund, and C. R. Taylor, "Mechanical work in terrestrial locomotion: Two basic mechanisms for minimizing energy expenditure," American Journal Physiology, vol. 233, no. 5, pp. R243R261, 1977.

[7] R. M. Alexander, "Three uses for springs in legged locomotion," The International Journal of Robotics Research, vol. 9, no. 2, pp. 53-61, April 1990.

[8] C. T. Motirtz and C. T. Farley, "Human hopping on damped surfaces: Strategies for adjusting leg mechanics," in Transactions of the Royal Society of London, 2003.

[9] D. P. Ferris, K. Liang, and C. T. Farley, "Runners adjust leg stiffness for their first step on a new running surface," Journal of Biomechanics, pp. 787-974, 1999.

[10] A. Duncan and M. J. N. McDonagh, "Stretch reflex distinguished from pre-programmed muscle activations following impacts in man," Journal of Physiology, vol. 526, pp. 457-468, 2000.

[11] T. Horita, P. V. Komi, C. Nicol, and H. Kyrolainen, "Interaction between pre-landing activities and stiffness regulation of the knee joint musculoskeletal system in the drop jump: implications to performance," European Journal of Applied Physiology, vol. 88, pp. 76-84, 2002.

[12] C. T. Farley, H. H. P. Houdijk, C. V. Strien, and M. Louie, "Mechanism of leg stiffness adjustment for hopping on surfaces of different stiffnesses," The American Physiological Society, pp. 1044-1055, 1998.

[13] T. M. Kubow and R. J. Full, "The role of the mechanical system in control; a hypothesis of self-stabilization in hexapedal runners," Phil. Trans. R. Soc. Lond., 1999.

[14] M. H. Raibert and J. K. Hodgins, Biological Neural Networks in Invertebrate Neuroethology and Robotics. Academic Press, 1993, ch. 14: Legged Robots, pp. 319-354. 